\title{
Magnetic and surface plasmon resonances for periodic lattices of plasmonic split-ring resonators
}

\author{
Ruey-Lin Chern* \\ Institute of Applied Mechanics, National Taiwan University, Taipei 106, Taiwan, Republic of China \\ (Received 31 January 2008; revised manuscript received 9 July 2008; published 12 August 2008)
}

\begin{abstract}
In this study, the author investigates magnetic and surface plasmon resonances for periodic lattices of plasmonic split-ring resonators by the use of the interface matching method. Magnetic resonance is characterized by a special frequency branch and illustrated with the localized eigenmode patterns. The basic features associated with magnetic resonance are present at the long wavelength as well as at the Bloch wavelength comparable to the size of a unit cell with minor distinctions. Near the surface plasma frequency, a large number of highly resonant branches appear and surface plasmon resonance gradually becomes the dominated feature. By varying the ratio of lattice constant to plasma wavelength, the influence of plasmonic property on magnetic resonance is examined for rather small and large skin depths.
\end{abstract}

DOI: 10.1103/PhysRevB.78.085116 PACS number(s): 78.67. - n, 78.20.Bh, 42.79. - e, 42.25. - p

\section{INTRODUCTION}

Split-ring resonator ${ }^{1}$ has been the essential element in the design of electromagnetic metamaterials ${ }^{2}$ in recent years. Intensive works were devoted to the studies of various splitring structures. ${ }^{3-6}$ Due to the adverse magnetic response, ${ }^{7-9}$ split-ring structures may exhibit a negative effective permeability (at the long wavelength), ${ }^{1,10}$ which is not available in naturally occurring materials. This unusual property is useful in building a composite medium with simultaneously negative permeability and permittivity ${ }^{11}$ and to demonstrate a negative index of refraction. ${ }^{12,13}$ Based on split-ring resonators, left-handed coplanar waveguides, ${ }^{14}$ low-power microwave plasma sources, ${ }^{15}$ and compact band pass filters ${ }^{16}$ can also be realized. In fact, the split ring is itself a high $Q$ resonator $^{17}$ and has been used in the design of electron-spin resonance spectroscopy ${ }^{18}$ and in measuring the microwave surface resistance. ${ }^{19}$

Magnetic response of the split-ring structure is attributed to resonance $e^{20,21}$ in which the ring acts as an inductor and the gap as a capacitor. The split-ring element is therefore considered an analog of the $L C$ circuit and serves to induce the magnetic activity in a structure composed of nonmagnetic materials. In order to seek for a large effect, very inhomogeneous fields inside the structure are required, ${ }^{1}$ which indicates that the currents and charges would be significant when the electromagnetic waves approach the resonance condition and have strong interactions with the medium.

For plasmonic split-ring structures, surface plasmon resonance serves as another distinguished feature. This type of resonance comes from collective excitation of electric charges in the plasmonic material coupled with the electromagnetic fields. As the frequency approaches the surface plasma frequency $\omega_{\mathrm{sp}}$, surface plasmon resonance may occur. The two types of resonances associated with the plasmonic split-ring structure result from the interaction of waves with the medium, which is usually described in terms of dispersion. As split-ring elements are arranged to form a periodic lattice, the resonance features are contained in the dispersion relations $\omega=\omega(\mathbf{k})$ in which the variations of electromagnetic fields in time and in space are correlated.

In periodic lattices, two scales of resonances are embedded in the overall dispersion characteristics: site resonance and lattice resonance. ${ }^{22}$ The former refers to the local response of an individual element and the latter to the system behavior due to the periodicity of the structure. At the long wavelength, the local response dominates the dispersion characteristics and can be well studied by the quasistatic approximation. ${ }^{1,23}$ At the Bloch wavelength comparable to the size of a unit cell, the local response and system behavior are intermixed to cause a somewhat complex phenomenon. A more general analysis would involve a link between the local and system responses. ${ }^{24}$

For plasmonic materials, the free-electron motion accounts for the dispersion and gives rise to frequency dependence of the dielectric property. The underlying problem is formulated as an eigensystem with the frequency as unknown. Due to the evanescent nature of electromagnetic field in plasmonic materials, the problem demands a delicate treatment of boundary conditions at the interface. The interface matching method is proposed for this purpose. This approach is aimed to resolve the fields and related physical quantities according to their surface-linking nature. On one hand, the fields may exhibit a drastic change or rapid variation across the interface at which they are nondifferentiable or even discontinuous. A straightforward manner to deal with the problem may lead to a nonlinear problem ${ }^{25}$ accompanied with unstable or even inaccurate solutions. On the other hand, the dominated physical quantities (such as surface charge) are closely related to the property of interface. ${ }^{26}$ The matching process at the interface brings in a linear formulation that can be dealt with standard eigenvalue solvers.

In this study, magnetic and surface plasmon resonances for plasmonic split-ring structures are investigated with emphasis on the dispersion characteristics. The frequency branch associated with magnetic resonance is identified and compared with the acoustical and optical branches that occur for most periodic structures. Basic features of magnetic resonance are illustrated with the corresponding localized eigenmode patterns in terms of fields, currents, charges, magnetic moment, and effective permeability. On one hand, the properties based on the effective-medium theory ${ }^{1}$ are recaptured and integrated together to depict the magnetic resonance from several aspects based on a more rigorous theory. On the other hand, magnetic resonance is explored at the conditions other than the long wavelength. In particular, basic features 
of magnetic resonance are identified at the Bloch wavelength comparable to the size of a unit cell. Their distinctions are examined on the frequency branch, mode pattern, and the band edges.

In another aspect, the distinguished feature of surface plasmon resonance is manifested on a large number of highly resonant branches around the surface plasma frequency $\omega_{\mathrm{sp}}$. Due to the strong coupling of photons and electrons, the resonant branches become dispersionless or insensitive to the change in Bloch wave vector. These branches may spread a rather wide range of frequency owing to the effective interaction of surface modes on the split-ring geometry. The freeelectron motion also has the influence upon magnetic resonance to some extent. This effect is studied by varying the plasmonic parameter defined as the ratio of lattice constant to plasma wavelength. For a small parameter, the skin depth is relatively large (compared to the size of split ring) and the plasmonic behavior on magnetic resonance has the largest effect. For a large parameter, the skin depth is relatively small and the effect of plasmonic behavior becomes negligible.

\section{INTERFACE MATCHING METHOD}

The interface matching method is designed to solve the eigenvalue problem for dispersive materials when the frequency is considered as unknown. ${ }^{26}$ This approach is particularly useful in resolving the plasmon-dominated problem, where surface modes are essential to the overall dispersion characteristics. The basic idea of the interface matching method is to separately deal with the wave equations in the strict insides of the dielectric and plasmonic materials and connect the two equations by matching the boundary condition at the interface. Equations in the separate regions (dielectric and plasmonic) as well as at the interface are combined together to yield a mixed formulation. The original nonlinear problem ${ }^{25}$ is recast into a linear eigensystem with standard format and can be efficiently solved by standard algorithms. ${ }^{27,28}$ A similar approach based on an auxiliary interfacial variable has been proposed to deal with the problems of plasmonic crystals, ${ }^{29}$ polaritonic crystals, ${ }^{30}$ and negative index crystals. ${ }^{31}$ In the present approach, the interfacial variable is not applied and the problem is formulated with a more direct manner.

Consider a periodic lattice of SRR columns with constant geometry along the axis normal to the lattice plane. Based on Maxwell's equations, the time-harmonic electromagnetic modes (with time dependence $e^{-i \omega t}$ ) are described in terms of the field component along the column axis as ${ }^{32}$

$$
\begin{gathered}
-\frac{1}{\varepsilon} \nabla^{2} E=\left(\frac{\omega}{c}\right)^{2} E, \\
-\nabla \cdot\left(\frac{1}{\varepsilon} \nabla H\right)=\left(\frac{\omega}{c}\right)^{2} H,
\end{gathered}
$$

for transverse magnetic (TM) and transverse electric (TE) polarizations, respectively. Here, TM (TE) is referred to the case where the $H(E)$ field lies completely on the SRR plane.

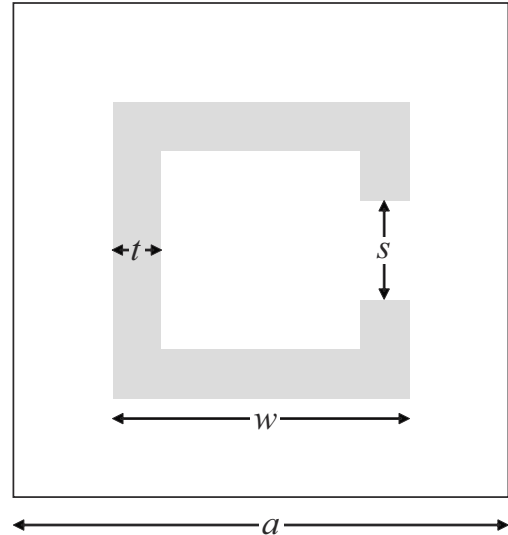

FIG. 1. Schematic of the unit cell for a periodic lattice of square split rings where $a$ is the lattice constant, $w$ is the ring width, $t$ is the ring thickness, and $s$ is the gap distance. The shaded region corresponds to the plasmonic material and the surrounding area to the dielectric.

For periodic structures with infinite extent, it is sufficient to solve the problem in one unit cell along with the Bloch condition,

$$
\phi\left(\mathbf{r}+\mathbf{a}_{i}\right)=e^{i \mathbf{k} \cdot \mathbf{a}_{i}} \phi(\mathbf{r}),
$$

applying at the unit-cell boundary where $\phi$ is either $E$ or $H$ field, $\mathbf{k}$ is the Bloch wave vector, and $\mathbf{a}_{i}(i=1,2)$ are the lattice translation vectors. The schematic of the unit cell is shown in Fig. 1. Using the Drude model $\varepsilon(\omega)=1-\omega_{p}^{2} /\left(\omega^{2}\right.$ $+i \omega \gamma)$ as the dielectric function of the metal and $\varepsilon_{d}$ as the dielectric constant of the background material, Eqs. (1) and (2) are formulated as eigensystems with the frequency $\omega$ as unknown, where $\omega_{p}$ is the bulk plasma frequency and $\gamma$ is the damping constant. As $\omega$ appears in the differential operator, the eigensystems in Eqs. (1) and (2) become nonlinear in frequency. ${ }^{25}$

The first step is to deal with the equations in the strict insides of the two different media (where the interface is excluded) so that the equation in either region can be rearranged as

$$
\begin{gathered}
-\frac{1}{\varepsilon_{d}} \nabla^{2} \phi=\left(\frac{\omega}{c}\right)^{2} \phi, \\
{\left[-\nabla^{2}+\left(\frac{\omega_{p}}{c}\right)^{2}\right] \phi=\left(\frac{\omega}{c}\right)^{2} \phi,}
\end{gathered}
$$

for $\phi$ in the dielectric and metal regions, respectively. The above two equations are then connected by matching the boundary conditions at the interface. Continuity of the tangential $H$ and $E$ fields across the interface gives

$$
\left[\frac{\partial E}{\partial n}\right]_{S}=0, \quad\left[\frac{1}{\varepsilon} \frac{\partial H}{\partial n}\right]_{S}=0,
$$

where $[\cdots]_{S}$ is the jump across the interface $S$. The equations in the strict insides [Eqs. (4) and (5)] as well as at the interface $[$ Eq. (6)] are arranged together to yield a generalized linear eigensystem that can be efficiently solved by standard 


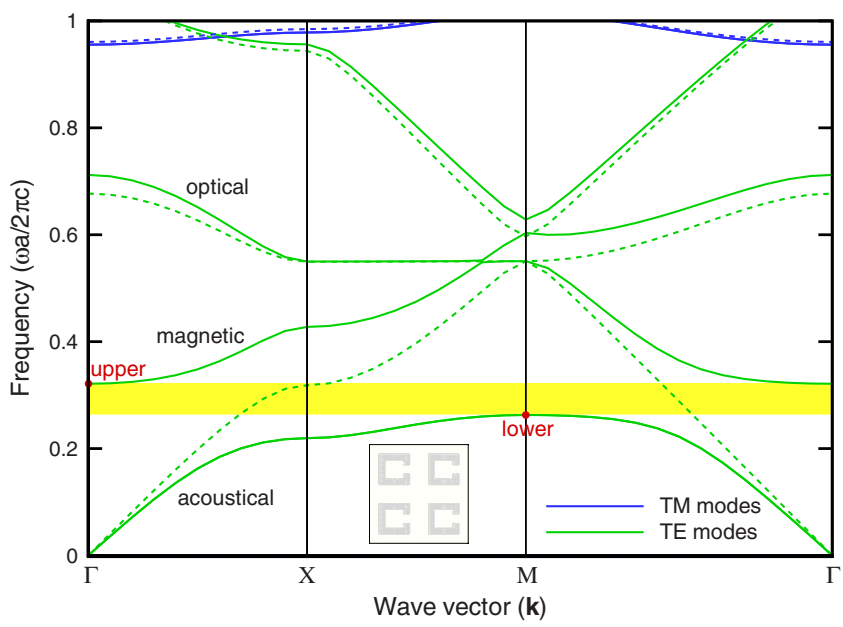

FIG. 2. (Color online) Dispersion relations for the periodic lattice of plasmonic split rings with $w / a=0.6, t / a=0.1, s / a=0.2$, and $a / \lambda_{p}=10$. The shaded region corresponds to the complete band gap and the left and right circular dots denote the upper and lower band edges, respectively. The inset shows four $(2 \times 2)$ unit cells of the structure. Dashed lines are results for the closed ring structure $(s$ $=0)$ with the same thickness and width.

eigenvalue solvers without resorting to nonlinear techniques. Details of the interface matching method can be found in Ref. 26.

\section{RESULTS AND DISCUSSION}

Both the magnetic and surface plasmon resonances occur in TE polarization, where the magnetic field aligns normal to the lattice plane. This is because for TM polarization, the electric fields are parallel to the metal surface and continuous across the interface between the dielectric and plasmonic materials. As a result, no surface charges exist to excite the resonances. For plasmonic materials, the plasma wavelength $\lambda_{p}=2 \pi / k_{p}=2 \pi c / \omega_{p}$ is a characteristic length scale associated with the free-electron oscillation and is also a measure of the skin depth for electromagnetic field. ${ }^{33}$ In the periodic structure with lattice constant $a$, the ratio $a / \lambda_{p}=\omega_{p} a / 2 \pi c$ then serves as a plasmonic parameter to describe the significance of plasmonic behavior. The dielectric constant $\varepsilon_{d}$ of the surrounding dielectric is unity. Unless specified, the damping constant $\gamma$ is assumed to be zero.

\section{A. Magnetic resonance}

\section{Dispersion relation}

Figure 2 shows the dispersion relations $\omega=\omega(\mathbf{k})$ for the periodic lattice of plasmonic split rings with $w / a=0.6, t / a$ $=0.1$, and $s / a=0.2$. The plasmonic parameter is given as $a / \lambda_{p}=10$ so that the ring thickness is comparable to the skin depth; that is, $t / \lambda_{p}=1$. The first and the third TE modes display a common feature for most periodic structures. They are qualitatively similar to the acoustical- and optical-phonon branches in the two-atoms crystal. ${ }^{34}$ The acoustical branch has a relatively lower frequency range, starting from zero frequency at the point $\Gamma[\mathbf{k}=(0,0)]$, growing up linearly with
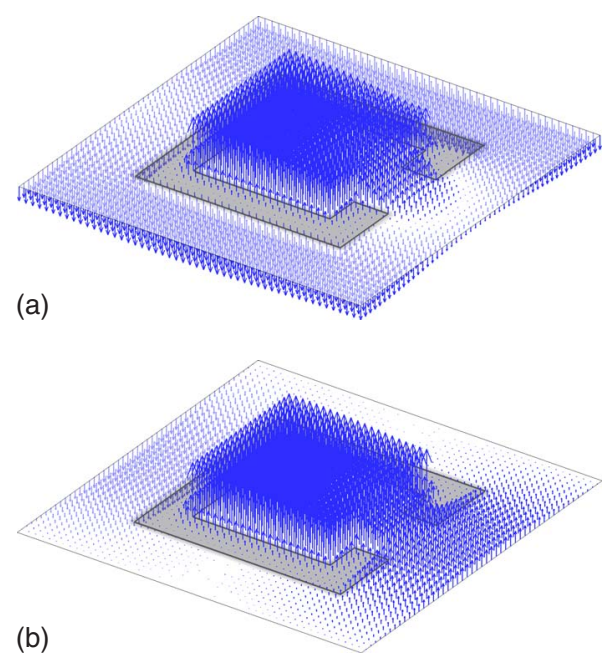

FIG. 3. (Color online) Magnetic-field vectors $(\operatorname{Re}[\mathbf{H}])$ of the TE eigenmodes for the plasmonic split-ring structure in Fig. 2. (a) the second mode at the point $\Gamma$ (upper band edge) and (b) the first mode at the point $M$ (lower band edge).

increasing the Bloch wave number $k$ and becoming flattened near the point $X[\mathbf{k}=(\pi / a, 0)]$ or $M[\mathbf{k}=(\pi / a, \pi / a)]$. On the other hand, the optical branch begins with a cut-off frequency and goes down as $k$ increases in the range $\Gamma-X$ or $\Gamma-M$.

\section{Resonance branch}

Between the acoustical and optical branches, a special TE frequency branch (the second band) appears, which is attributed to magnetic resonance associated with the split-ring structure. For comparison, the dispersion curves for the closed ring structure $(s=0)$ with the same width and thickness are overlaid in Fig. 2 (in dashed lines) in which the resonance branch is absent. Basic features related to the resonance branch will be identified from several aspects including the field pattern, current and charge distribution, resultant magnetic moment, and effective permeability. The resonance branch begins with a cut-off frequency (substantially lower than that for the optical branch) and draws near to the optical branch as $k$ increases. A complete band gap is opened between the acoustical and resonance branches.

\section{Localized modes}

In Fig. 3(a), the eigenmode on the resonance branch at the point $\Gamma$ is illustrated with the magnetic-field vectors. The fields are intense inside the split ring, while outside the field amplitude is substantially reduced and the field orientation is even reversed. This inhomogeneous field pattern depicts a local response of the individual element where the fields are concentrated inside the split ring and the interaction between adjacent elements is weak. Localization of the fields within individual elements prevents the electromagnetic energy from being transferred across the unit cells and indicates the existence of a complete band gap in the dispersion diagram (cf. Fig. 2). This feature consists with the negative value of the effective permeability ${ }^{1}$ and the low transmission in the corresponding frequency range. .1,35,36 $^{-3}$ 


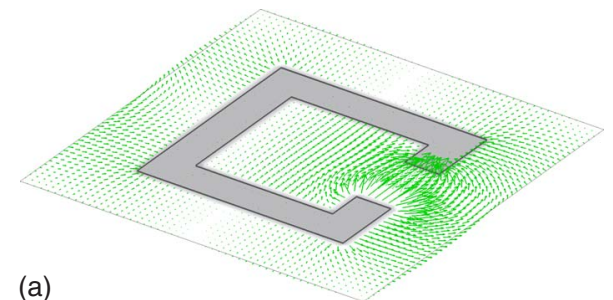

(a)

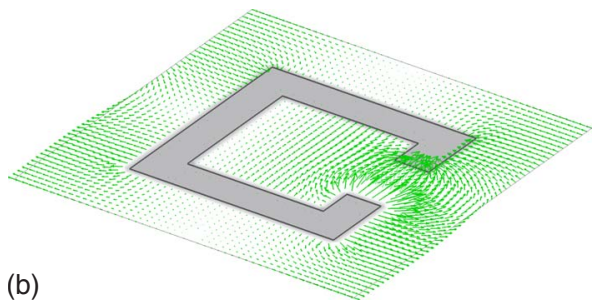

FIG. 4. (Color online) Electric-field vectors $(\operatorname{Im}[\mathbf{E}])$ of the TE eigenmodes for the plasmonic split-ring structure in Fig. 2: (a) upper and (b) lower band edges, respectively.

\section{Band edges}

The point $\Gamma$ on the resonance branch [where the eigenmode in Fig. 3(a) is shown] corresponds to the upper band edge and is considered as the long-wavelength condition. As the Bloch wave number $k$ increases, the resonance branch gradually deviates from the band gap and begins to loses its magnetic-resonance nature. Meanwhile, the acoustical branch gradually approaches the lower band edge at the point $M$. This is the condition where the Bloch wavelength is comparable to the size of a unit cell. The corresponding eigenmode in Fig. 3(b) shows a similar field pattern within the unit cell, except that the fields outside the split ring have an antisymmetric distribution on opposing sides, in contrast to the symmetric pattern in Fig. 3(a). This difference comes from the phase modulation by the Bloch wave vector and is consistent with the Bloch condition [Eq. (3)] that has to be satisfied at the unit-cell boundary. As the fields are intense inside the split ring, the localized nature associated with magnetic resonance still appears with a difference of the resonance frequency. The frequency difference between the long wavelength (resonance branch at the point $\Gamma$ ) and the Bloch wavelength comparable to a unit cell (acoustical branch at the point $M$ ) makes up the band-gap width caused by magnetic resonance.

\section{Electric fields}

The inhomogeneous magnetic-field distributions (Fig. 3) accompany localized electric-field patterns as shown in Fig. 4. The electric fields are calculated according to AmpereMaxwell's law $\nabla \times \mathbf{H}=-i \omega \varepsilon \mathbf{E}$ as

$$
\mathbf{E}=\frac{i}{\omega \varepsilon}\left(\frac{\partial H_{z}}{\partial y} \hat{\mathbf{x}}-\frac{\partial H_{z}}{\partial x} \hat{\mathbf{y}}\right) .
$$

As the magnetic fields have significant changes from inside the split ring to outside, the electric fields in the gap region (the passage between inside and outside) are strongly enhanced. The electric energy is raised and a mechanism of

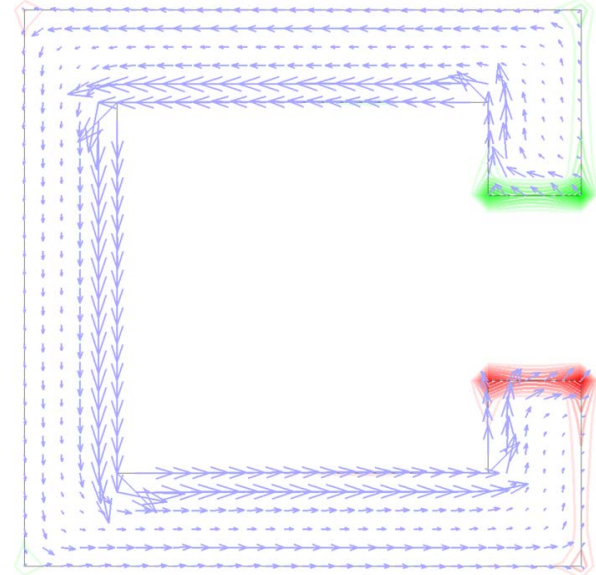

(a)

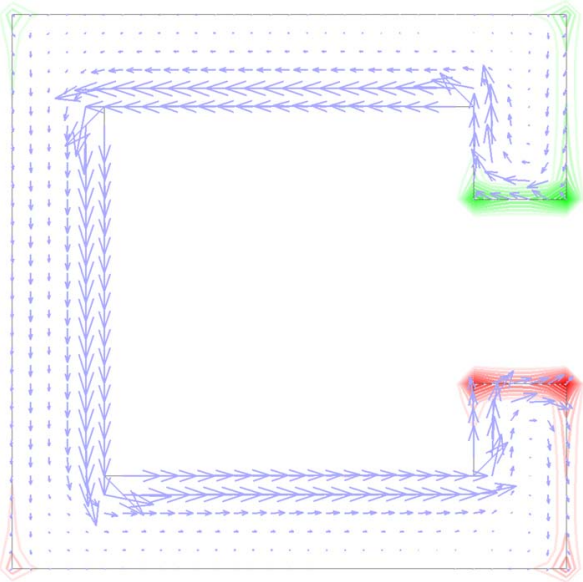

(b)

FIG. 5. (Color online) Surface charge strengths $(\operatorname{Im}[\sigma])$ and polarization current vectors $\left(\operatorname{Re}\left[\mathbf{J}_{p}\right]\right)$ of the TE eigenmodes for the plasmonic split-ring structure in Fig. 2: (a) upper and (b) lower band edges, respectively. The red (dark gray) and green colors (light gray) correspond to positive and negative charges, respectively.

energy conversion between the electric (stored in the gap) and magnetic energies (stored in the ring) has been initiated. Inhomogeneous as well as localized fields thus serve as the typical feature of magnetic resonance associated with the split-ring structure. Note that the distinction between Fig. 4(a) (upper band edge) and Fig. 4(b) (lower band edge) becomes less significant except at the portion near the cell boundary.

\section{Surface charges}

Locally enhanced electric fields (Fig. 4) give rise to substantial surface charges accumulated on the gap edges as shown in Fig. 5. The surface charge is related to the discontinuity of normal electric field at the surface as $\sigma$ $=\varepsilon_{0}[\hat{\mathbf{n}} \cdot \mathbf{E}]_{S}$, where $[\cdots]_{S}$ denotes the jump across the interface $S$ and $\hat{\mathbf{n}}$ is the unit vector normal to the interface. As the electric fields for the upper and lower band edges [Figs. 4(a) and 4(b)] share a very similar pattern near the interface, the surface charges shown in Figs. 5(a) and 5(b) are nearly identical. Accumulation of surface charges illustrates the function 
of a capacitor imbedded in the split-ring element as magnetic resonance occurs.

\section{Polarization currents}

In another aspect, the polarization fields $\mathbf{P}=\left(\varepsilon-\varepsilon_{0}\right) \mathbf{E}$ induced in the plasmonic split-ring structure produce the polarization currents $\mathbf{J}_{p}=\frac{\partial \mathbf{P}}{\partial t}$. These volume currents circulate along the split ring and give rise to a magnetic moment (per unit length in the direction of $\mathbf{H})$ as $\mathbf{m}=\frac{1}{2} \oint \mathbf{r} \times \mathbf{J}_{p} d a{ }^{37}$ As most current vectors orient in the same (counterclockwise) sense, a considerable (positive) magnetic moment is attained. Considering the periodic structure as a homogeneous medium, an average magnetization (per unit length) $\mathbf{M}=\frac{\mathbf{m}}{a^{2}}$ is then associated with the unit cell. This feature illustrates the function of an inductor imbedded in the split-ring element as magnetic resonance occurs.

\section{Effective permeability}

According to the effective-medium theory proposed by Pendry et al., ${ }^{1}$ the effective permeability is described under the long-wavelength assumption in terms of the average fields as $\mu_{\mathrm{eff}}=\frac{B_{\text {ave }}}{\mu_{0} H_{\text {ave }}}$, where the subscript "ave" denotes the field averaging - area averaging for $B_{\text {ave }}$ and line averaging for $H_{\text {ave }}$. Due to the depolarization fields, ${ }^{1}$ the average magnetic flux over the unit cell for a periodic lattice of infinite extent will not change. Positive magnetization thus reduces the magnetic-field intensity as $H_{\text {ave }}=\frac{B_{\text {ave }}}{\mu_{0}}-M$. Here, $H_{\text {ave }}$ corresponds to the field outside the split ring in order to ensure the continuity of tangential $H_{\text {ave }}$ along the cell boundary. ${ }^{1}$ This feature is also manifested on the magnetic-field patterns (Fig. 3) where the field amplitude outside the split ring is substantially reduced and the field orientation is even reversed. Accordingly, the effective permeability is related to $M$ as $\mu_{\text {eff }}=\frac{B_{\text {ave }}}{B_{\text {ave }}-\mu_{0} M},{ }^{38}$ which could be negative if $M$ is sufficiently large. This feature states that for structures made of nonmagnetic materials (without intrinsic magnetism), a large induced magnetization (due to resonance) may cause an adverse magnetic response.

\section{Distinctions}

Basic features related to magnetic resonance at the long wavelength appear at the Bloch wavelength comparable to the size of a unit cell as well. This is manifested on very inhomogeneous magnetic fields (Fig. 3), strongly enhanced electric fields (Fig. 4), accumulated surface charges and circulating currents (Fig. 5), considerable magnetic moment, and negative effective permeability. These features show that magnetic resonance occurs over a frequency range with various wavelengths - a consequence of the coupling of individual magnetic resonance with the periodicity of the lattice. However, there are two distinctions associated with the latter condition. First, the magnetic resonance occurs on the acoustical branch instead of the localized branch. The resonance frequency is to some extent lower than that at the long wavelength. Second, the corresponding localized mode shows a slightly different field pattern outside the split ring due to the phase change across the unit cell.

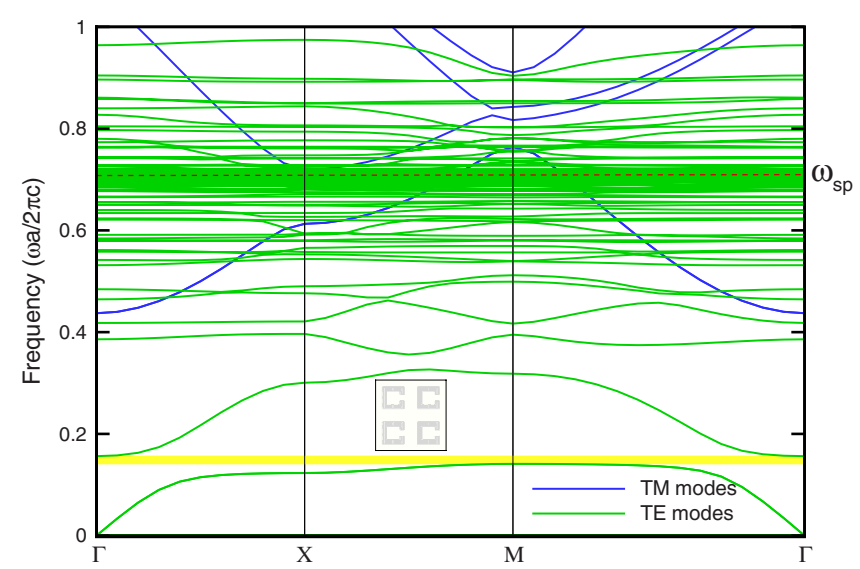

FIG. 6. (Color online) Dispersion relations for the plasmonic split-ring structure of the same geometry in Fig. 2 with $a / \lambda_{p}=1$. The dashed line indicates the surface plasma frequency $\omega_{\text {sp. }}$.

\section{B. Surface plasmon resonance}

\section{Surface plasmon branches}

Figure 6 shows the dispersion relations $\omega=\omega(\mathbf{k})$ for the plasmonic split-ring structure with the same geometry in Fig. 2. The plasmonic parameter is given as $a / \lambda_{p}=1$ so that the lattice constant is comparable to the skin depth. It is shown that a large number of TE frequency branches appear and intensively gather around the surface plasma frequency $\omega_{\mathrm{sp}}$ given by

$$
\omega_{\mathrm{sp}}=\frac{\omega_{p}}{\sqrt{1+\varepsilon_{d}}} .
$$

Note that $\omega_{\mathrm{sp}}$ has the same form as that in the planar surfaces. ${ }^{39}$ These branches display a highly resonant nature due to the appearance of surface plasmons, which come from the collective excitation of electric charges in the plasmonic material. As the frequency approaches $\omega_{\mathrm{sp}}$, more resonant branches emerge within a very narrow frequency interval. This is a typical feature of high degeneracy associated with surface plasmon resonance in the two-dimensional lattice.

Due to the strong coupling of photons and electrons, surface plasmon branches become insensitive to the change Bloch wave vector. As a result, the resonant branches tend to be flattened or dispersionless, which is more evident as the frequency gets closer to $\omega_{\mathrm{sp}}$. In another aspect, the interaction of surface modes on both sides of the ring surface lifts the degeneracy and thus broadens the resonance frequency range. As a result, surface plasmon branches may spread over a rather wide range of frequency centered about $\omega_{\text {sp. }}$. Furthermore, the interface between the dielectric and plasmonic materials represents a closed surface in the lattice plane (cf. Fig. 1), which can sustain as many variation patterns as possible and, therefore, an infinite number of surface plasmon modes are expected to exist. ${ }^{40}$ The above three features (band flattening, band broadening, and an infinite number of surface modes) bear a close resemblance to those in plasmonic crystals. ${ }^{41}$ 


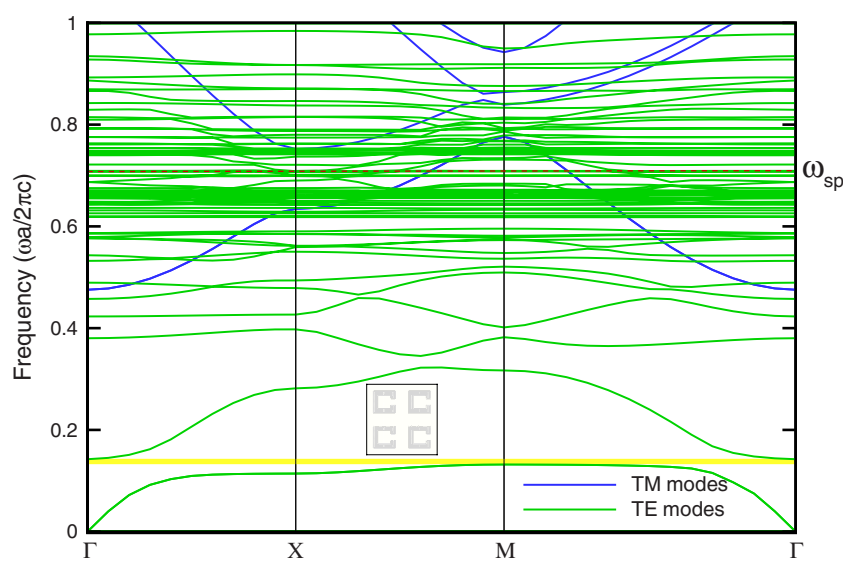

FIG. 7. (Color online) Dispersion relations for the plasmonic split-ring structure of the same geometry in Fig. 2 with $a / \lambda_{p}=1$ and $\gamma / \omega_{p}=0.1$.

\section{Effect of loss}

If the intrinsic loss of metal is taken into account, the resonance branches may alter some characteristics. Figure 7 shows the dispersion relation for the same structure in Fig. 6 with $\gamma / \omega_{p}=0.1$. The resonance branches become broadened around the surface plasma frequency $\omega_{\text {sp }}$ and the strong degeneracy was lifted to some extent. This phenomenon is attributed to the reduction in symmetry for degenerate modes in the presence of damping, which is particularly evident when the loss becomes dominated.

\section{Surface plasmon modes}

Figure 8 shows the magnetic-field vectors $(\operatorname{Re}[\mathbf{H}])$ and electric-field vectors $(\operatorname{Im}[\mathbf{E}])$ of a typical surface plasmon mode near $\omega_{\text {sp }}$ for the same structure in Fig. 6. The surface plasmon mode pattern displays an even more localized nature (than that for magnetic resonance) with the fields not only localized within the individual unit cell but also confined at the metal surface. As the excitation of free electrons
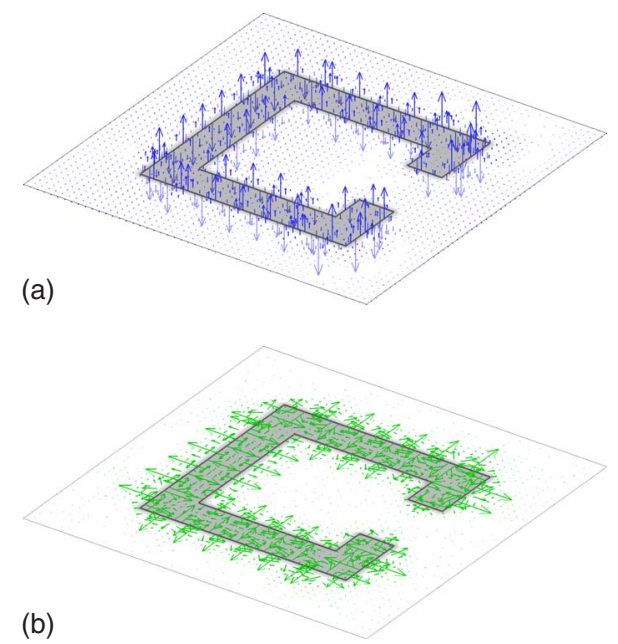

FIG. 8. (Color online) (a) Magnetic-field vectors $(\operatorname{Re}[\mathbf{H}])$ and (b) electric-field vectors $(\operatorname{Im}[\mathbf{E}])$ of a typical surface plasmon mode for the plasmonic split-ring structure in Fig. 6. prevents most of the fields from penetrating into the plasmonic material, the fields of surface plasmon mode become highly localized at the interface and rapidly decay in either the dielectric or plasmonic material. ${ }^{41}$ This feature consists with the dispersionless property of surface plasmon branches that the change in Bloch wave vector has less effect on the dispersion of surface plasmons.

\section{Effect of plasmonic parameter}

Near the surface plasma frequency $\omega_{\text {sp }}$, surface plasmon resonance gradually becomes the dominated feature while magnetic resonance occurs at a relatively lower frequency range. In plasmonic materials, the magnetic-resonance frequency is not only determined by the split-ring geometry but also by the electron motion. This effect can be explored by altering the plasmonic parameter $a / \lambda_{p}$; the ratio of lattice constant to plasma wavelength. A small ratio corresponds to a relatively large skin depth (or relatively small structure) and indicates the significance of electron motion while a large ratio corresponds to a relatively small skin depth and indicates the significance of geometry property.

\section{Small ratio}

Figure 9(a) shows the variation of magnetic-resonance frequency range (in terms of upper and lower band edges) with the change in $a / \lambda_{p}$ while the split-ring geometry (width, thickness, and gap) is kept fixed. It is shown that magnetic resonance is shifted to higher frequencies (on the scale of $\left.\omega / \omega_{p}\right)$ as $a / \lambda_{p}$ is reduced, which is feasible as a small split ring may exhibit a higher resonance frequency. However, the frequency becomes saturated as $a / \lambda_{p}$ is sufficiently small. This feature shows that magnetic-resonance frequency is not scalable in plasmonic materials, which is attributed to the increasing importance of kinetic energy of free electrons. ${ }^{42}$

\section{Small size}

Figure 9(b) shows the variation of magnetic-resonance frequency range with the change in split-ring width $w / a$ (thickness $t / a$ and gap $s / a$ are changed accordingly) while $a / \lambda_{p}=0.1$ is kept fixed. The resonance frequency again reaches a saturated value at small $w / a$ where the frequencies of upper and lower band edges coincide and the band gap disappears.

\section{Large ratio}

In another aspect, Fig. 10 shows the variation of magnetic-resonance frequency range at large $a / \lambda_{p}$, which corresponds to relatively small skin depths. It is shown that magnetic-resonance frequency approaches an asymptotic frequency (on the scale of $\omega a / 2 \pi c$ ) at very large $a / \lambda_{p}$, which is identified as the result for perfect metal. In this situation, the plasmonic behavior becomes less important and magnetic resonance is mostly determined by the split-ring geometry.

\section{Magnetic moment}

Finally, the effect of plasmonic property is displayed with the dependence of magnetic moment on the plasmonic pa- 

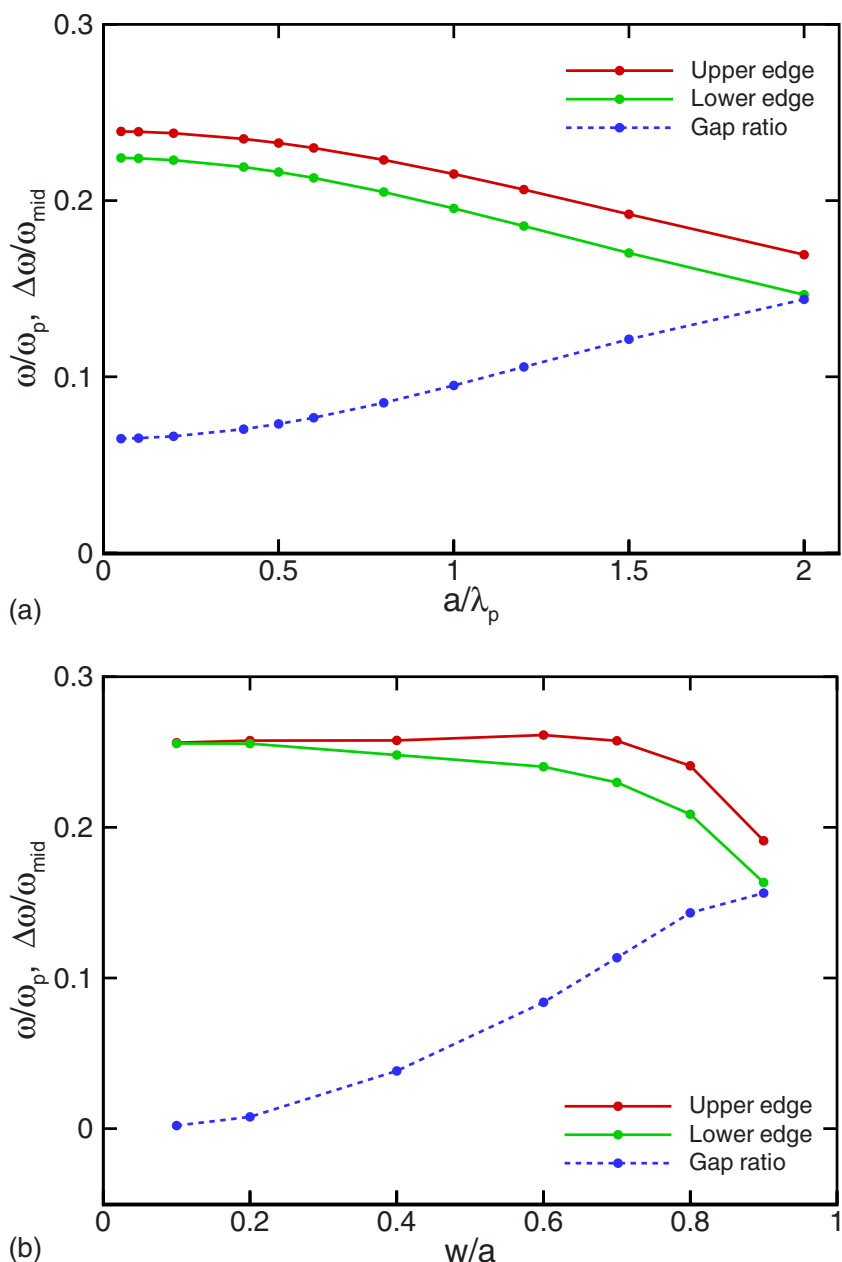

FIG. 9. (Color online) Variation of magnetic-resonance frequency range for the plasmonic split-ring structure (a) with fixed geometry: $w / a=0.5, t / a=0.1$, and $s / a=0.3$ for various $a / \lambda_{p}$ and (b) with fixed $a / \lambda_{p}=0.1$ for various $w / a$ ( $t / a$ and $s / a$ are changed accordingly).

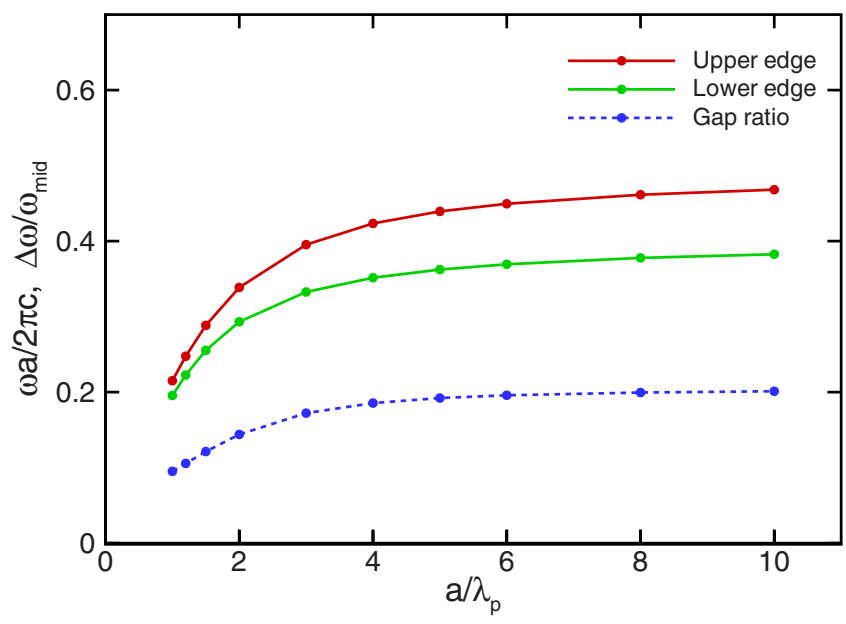

FIG. 10. (Color online) Variation of magnetic-resonance frequency range for the plasmonic split-ring structure with $w / a=0.5$, $t / a=0.1$, and $s / a=0.3$ for various $a / \lambda_{p}$.

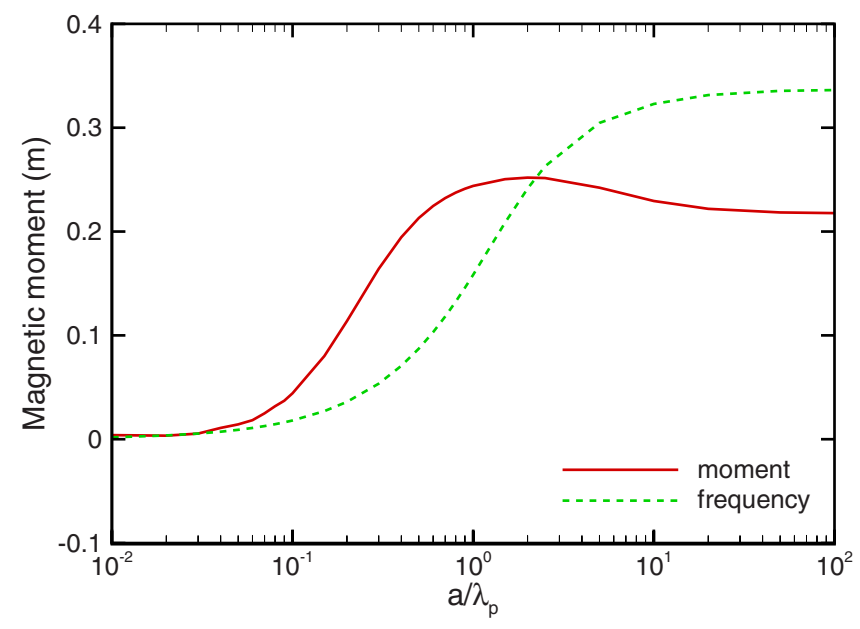

FIG. 11. (Color online) Dependence of magnetic moment on the plasmonic parameter $a / \lambda_{p}$ for the plasmonic split-ring structure with $w / a=0.6, t / a=0.1$, and $s / a=0.2$.

rameter. Figure 11 shows the variation of magnetic moment (at the upper band edge) with $a / \lambda_{p}$ (in logarithm scale) for the plasmonic split-ring structure with $w / a=0.6, t / a=0.1$, and $s / a=0.2$. For small $a / \lambda_{p}(<0.2)$, the magnetic moment increases linearly with the plasmonic parameter. In this range, the polarization current strengths are relatively weak. At large $a / \lambda_{p}(>10)$, the magnetic moment tends to approach a constant value where the currents are highly localized at the interface. A local maximum of the magnetic moment is located at an intermediate $a / \lambda_{p}(\approx 2.5)$. In this situation, the plasmonic property exhibits a most favorable contribution for sustaining the magnetic moment. Meanwhile, the magnetic-resonance frequency (in dashed line) increases monotonically with the plasmonic parameter until it is saturated.

\section{CONCLUDING REMARKS}

In conclusion, magnetic and surface plasmon resonances for periodic lattices of plasmonic split-ring resonators have been investigated. The interface matching method was proposed to solve the nonlinear eigenvalue problem (with the frequency as unknown) with a careful treatment of boundary conditions at the interface. Basic features of magnetic resonance are characterized by a special TE frequency branch (between the acoustical and optical branches) and well illustrated with the localized eigenmodes in terms of very inhomogeneous magnetic fields, strongly enhanced electric fields, accumulated surface charges, circulating polarization currents, considerable magnetic moment, and negative effective permeability. These features are present at the long wavelength as well as at the Bloch wavelength comparable to the size of a unit cell. Distinctions between the two situations were identified regarding the frequency branch and mode pattern.

In another aspect, surface plasmon resonance occurs as the frequency approaches the surface plasma frequency $\omega_{\text {sp }}$. The distinguished feature is the appearance of a large 
number of highly resonant branches gathered around $\omega_{\mathrm{sp}}$. Band flattening, band broadening, and expected infinite number of surface modes bear a close resemblance to those in plasmonic crystals. The influence of plasmonic property on magnetic resonance was also examined by altering the the ratio of lattice constant to plasma wavelength. For small ratios (relatively large skin depths), the kinetic energy of free electrons increases its importance and the resonance frequency reaches a saturated value. For large ratios (relatively small skin depths), the resonance frequency approaches that for the prefect metal structure and magnetic resonance is mostly determined by geometric property.

\section{ACKNOWLEDGMENTS}

The author thanks Ding-Ping Tsai at National Taiwan University and Pi-Gang Luan at National Central University for their helpful discussions. This work was supported in part by National Science Council of the Republic of China under Contracts No. NSC 96-2221-E-002-190-MY3 and No. NSC 96-2120-M-002-017. *chern@iam.ntu.edu.tw

${ }^{1}$ J. B. Pendry, A. J. Holden, D. J. Robbins, and W. J. Stewart, IEEE Trans. Microwave Theory Tech. 47, 2075 (1999).

${ }^{2}$ D. R. Smith, J. B. Pendry, and M. C. K. Wiltshire, Science 305, 788 (2004).

${ }^{3}$ D. Schurig, J. J. Mock, and D. R. Smith, Appl. Phys. Lett. 88, 041109 (2006).

${ }^{4}$ C. Rockstuhl, T. Zentgraf, H. Guo, N. Liu, C. Etrich, I. Loa, K. Syassen, J. Kuhl, F. Lederer, and H. Giessen, Appl. Phys. B: Lasers Opt. 84, 219 (2006).

${ }^{5}$ Y. Liu, N. Fang, D. Wu, C. Sun, and X. Zhang, Appl. Phys. A: Mater. Sci. Process. 87, 171 (2007).

${ }^{6}$ N. Liu, H. Guo, L. Fu, S. Kaiser, H. Schweizer, and H. Giessen, Nat. Mater. 7, 31 (2008).

${ }^{7}$ T. J. Yen, W. J. Padilla, N. Fang, D. C. Vier, D. R. Smith, J. B. Pendry, D. N. Basov, and X. Zhang, Science 303, 1494 (2004).

${ }^{8}$ S. Linden, C. Enkrich, M. Wegener, J. Zhou, T. Koschny, and C. Soukoulis, Science 306, 1351 (2004).

${ }^{9}$ N. Katsarakis, G. Konstantinidis, A. Kostopoulos, R. S. Penciu, T. F. Gundogdu, M. Kafesaki, E. N. Economou, T. Koschny, and C. M. Soukoulis, Opt. Lett. 30, 1348 (2005).

${ }^{10}$ A. Ishikawa, T. Tanaka, and S. Kawata, Phys. Rev. Lett. 95, 237401 (2005).

${ }^{11}$ D. R. Smith and N. Kroll, Phys. Rev. Lett. 85, 2933 (2000).

${ }^{12}$ R. A. Shelby, D. R. Smith, and S. Schultz, Science 292, 77 (2001).

${ }^{13}$ C. G. Parazzoli, R. B. Greegor, K. Li, B. E. C. Koltenbah, and M. Tanielian, Phys. Rev. Lett. 90, 107401 (2003).

${ }^{14}$ F. Martın, Appl. Phys. Lett. 83, 4652 (2003).

${ }^{15}$ F. Iza and J. Hopwood, IEEE Trans. Plasma Sci. 31, 782 (2003).

${ }^{16}$ F. Falcone, F. Martin, J. Bonache, R. Marques, T. Lopetegi, and M. Sorolla, IEEE Microw. Wirel. Compon. Lett. 14, 10 (2004).

${ }^{17}$ W. N. Hardy and L. A. Whitehead, Rev. Sci. Instrum. 52, 213 (1981).

${ }^{18}$ F. Momo, A. Sotgiu, and R. Zonta, J. Phys. E 16, 43 (1983).

${ }^{19}$ D. A. Bonn, D. Morgan, and W. N. Hardy, Rev. Sci. Instrum. 62, 1819 (1991).

${ }^{20}$ P. Gay-Balmaz and O. J. F. Martin, J. Appl. Phys. 92, 2929 (2002).

${ }^{21}$ J. García-García, F. Martín, J. D. Baena, R. Marqués, and L.
Jelinek, J. Appl. Phys. 98, 033103 (2005).

${ }^{22}$ F. J. García de Abajo, J. J. Sáenz, I. Campillo, and J. S. Dolado, Opt. Express 14, 7 (2006).

${ }^{23}$ C. R. Simovski, P. A. Belov, and S. He, IEEE Trans. Antennas Propag. 51, 2582 (2003).

${ }^{24}$ R. Liu, T. J. Cui, D. Huang, B. Zhao, and D. R. Smith, Phys. Rev. E 76, 026606 (2007).

${ }^{25}$ O. Toader and S. John, Phys. Rev. E 70, 046605 (2004).

${ }^{26}$ R. L. Chern, Phys. Rev. B 77, 045409 (2008).

${ }^{27}$ L. N. Trefethen and D. Bau, Numerical Linear Algebra (SIAM, Philadelphia, 1997).

${ }^{28}$ J. W. Demmel, Applied Numerical Linear Algebra (SIAM, Philadelphia, 1997).

${ }^{29}$ C. C. Chang, R. L. Chern, C. C. Chang, and R. R. Hwang, Phys. Rev. B 72, 205112 (2005).

${ }^{30}$ R. L. Chern, C. C. Chang, and C. C. Chang, Phys. Rev. B 73, 235123 (2006).

${ }^{31}$ R. L. Chern, C. C. Chang, and C. C. Chang, Phys. Rev. B 74, 155101 (2006).

${ }^{32} \mathrm{~W}$. Chew, Waves and Fields in Inhomogeneous Media (IEEE, New York, 1995).

${ }^{33}$ G. Shvets, and Y. A. Urzhumov, J. Opt. A, Pure Appl. Opt. 8, S122 (2006).

${ }^{34}$ C. Kittel, Introduction to Solid State Physics, 8th ed. (Wiley, New York, 2004).

${ }^{35}$ P. Markoš and C. M. Soukoulis, Phys. Rev. E 65, 036622 (2002).

${ }^{36}$ A. K. Azad, J. Dai, and W. Zhang, Opt. Lett. 31, 634 (2006).

${ }^{37}$ J. D. Jackson, Classical Electrodynamics, 3rd ed. (Wiley, New York, 1999).

${ }^{38}$ H. Chen, L. Ran, J. Huangfu, T. M. Grzegorczyk, and J. A. Kong, J. Appl. Phys. 100, 024915 (2006).

${ }^{39}$ H. Raether, Surface Plasmons on Smooth and Rough Surfaces and on Gratings (Springer, New York, 1988).

${ }^{40}$ T. Ito and K. Sakoda, Phys. Rev. B 64, 045117 (2001).

${ }^{41}$ R. L. Chern, C. C. Chang, and C. C. Chang, Phys. Rev. E 73, 036605 (2006).

${ }^{42}$ J. Zhou, T. Koschny, M. Kafesaki, E. N. Economou, J. B. Pendry, and C. M. Soukoulis, Phys. Rev. Lett. 95, 223902 (2005). 